\title{
Upgrade of the ALICE ITS in LS3
}

\author{
Magnus Mager* \\ CERN \\ E-mail: Magnus. Magerdcern.ch
}

\section{on behalf of the ALICE collaboration}

\begin{abstract}
ALICE is planning to replace its three innermost tracking layers with a fully cylindrical, bent silicon tracker during LHC Long Shutdown 3 (LS3, targeting 2024-25). The new detector will reach an unprecedented low material budget of below $0.05 \% X_{0}$ per layer, combined with an intrinsic spatial resolution below $5 \mu \mathrm{m}$ in $z$ - and $r \varphi$-directions. Its main building part is an ultra-thin $(20 \mu \mathrm{m}$ to $40 \mu \mathrm{m}$ ), wafer-scale $(300 \mathrm{~mm}$ ) CMOS Monolithic Active Pixel Sensor, that will be developed in $65 \mathrm{~nm}$ technology for this purpose. The sensor dimensions reach up to 280 by $94 \mathrm{~mm}$, and, owing to the flexible nature of silicon at these thicknesses, is bent into half-cylinders of radii of 18,24 , and $30 \mathrm{~mm}$, respectively, to form the new concentric layers.

This contribution addresses the detector R\&D road map as well as projected improvements in performance and related physics yields. The combination of reduced material budget, closer proximity to the interaction point, and high intrinsic resolution translate into a significant advancement in the measurement of short-lived particles and low-mass di-electrons, which are amongst the main physics goals of ALICE.
\end{abstract}

The 28th International Workshop on Vertex Detectors (VERTEX)

13-18 October 2019

Lafodia Sea Resort, Lopud Island, Croatia

${ }^{*}$ Speaker. 


\section{Introduction}

Currently, in the Long Shutdown 2 (LS2) of LHC, ALICE is upgrading its detector apparatus in several aspects to be able to collect events at a higher rate, as well as with higher resolution and precision. A central part of this upgrade is a new Inner Tracking System ("ITS2"), a large scale $\left(10 \mathrm{~m}^{2}\right)$ silicon detector, fully based on Monolithic Active Pixel Sensors (MAPS) [1, 2].

With MAPS technology, ITS2 will provide ALICE with unprecedented performance in terms of tracking resolution and reconstruction efficiency especially at low particle momenta $(\leq 1 \mathrm{GeV} / c)$. Here, the three features of closeness to interaction point $(23 \mathrm{~mm})$, intrinsic sensor resolution $\left(\sigma_{r \varphi} \approx\right.$ $\left.\sigma_{z} \approx 5 \mu \mathrm{m}\right)$ and low material budget $\left(\approx 0.3 \% X_{0}\right)$ are the key parameters that define the detector performance.

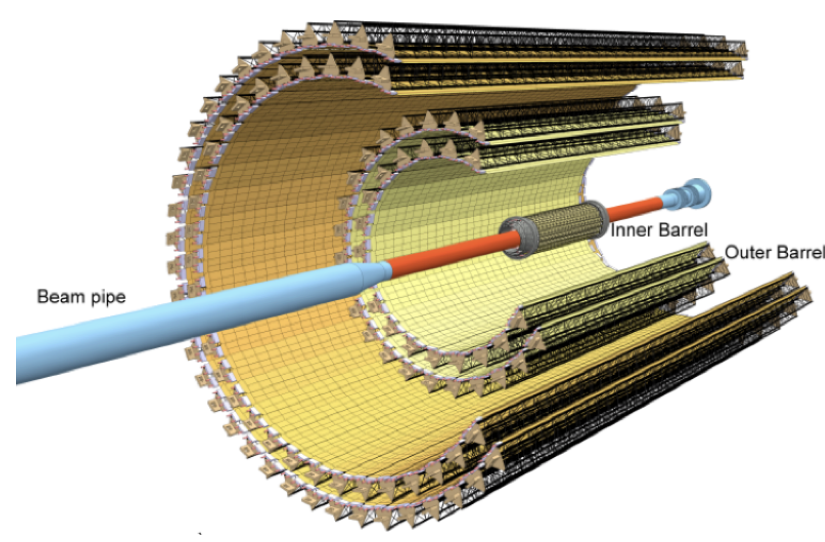

(a) full detector, with 7 layers

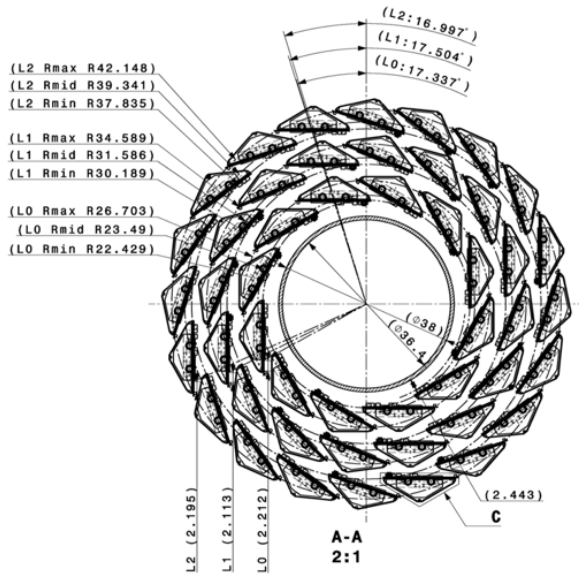

(b) close-up of the Inner Barrel (3 layers)

Figure 1: Layout of the ITS2, as being currently installed in LHC LS2. Figures from [1] with final dimensions.

Figure 1 illustrates the layout of the ITS2, shows its construction out of seven concentric layers, and details the layout of the Inner Barrel, which comprises the innermost three layers. While the detector is extremely lightweight, using carbon structures for its mechanical support, in particular Fig. $1 \mathrm{~b}$ gives an indication that the sensor itself makes up only for a very small fraction of the total detector.

\subsection{Material budget contributions}

More quantitatively, Fig. 2 gives the breakdown of material budget for the innermost layer of ITS2. Indeed, the sensor itself is responsible for only one seventh of the total amount, the rest being mechanical support, power and data distribution as well as cooling.

We believe that we can remove the need for all of these by employing wafer-scale ultra-thin CMOS sensors that are bent around the beam pipe.

The reasoning is as follows:

- Cooling: During the ITS2 R\&D, it was shown that we could base the cooling strategy on air cooling, if the power density would stay below $20 \mathrm{~mW} \mathrm{~cm}^{-2}$-a limit given by airflowinduced mechanical vibrations, which should stay far below the detector resolution. Looking 


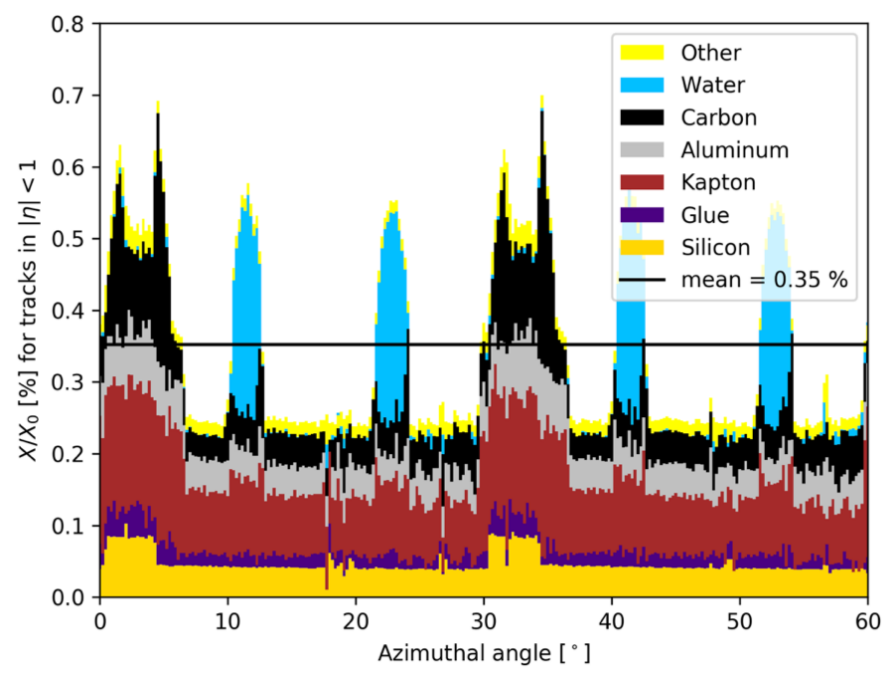

Figure 2: Material budget distribution and breakdown of the innermost ITS2 layer. Figure from [3].

at ALPIDE, which has a nominal power density of $40 \mathrm{~mW} \mathrm{~cm}^{-2}$, this is indeed already in reach: the pixel matrix of ALPIDE only consumes about $6 \mathrm{~mW} \mathrm{~cm}^{-2}$, the rest is dissipated at the digital periphery. On a wafer-scale sensor, we can arrange the periphery to stay outside the detector acceptance (where water cooling is available).

- Mechanical support: With the paper-like properties of ultra-thin $(20 \mu \mathrm{m}$ to $40 \mu \mathrm{m})$ silicon wafers, we plan to wrap the sensor around the beam pipe. This will stiffen the sensor dimensional. In addition, the distance of the planes will be kept using open-cell carbon foam, which is quasi-transparent for particles (radiation length $O(100 \mathrm{~m})$ ) and allows airflow.

- Power and signal distribution: The need of a flex-printed circuit board will cease, as there is no need anymore to interconnect several chips. Power distribution will become easier as the matrix consumes relatively little (see previous point), and for data, one largely benefits from the much higher integration density (line width) on a CMOS chip with respect to a printed circuit board.

While reasonable, all these items remain to be proven and quantified, which is the core of the upcoming $R \& D$ phase.

\section{Detector Concept and R\&D topics}

The proposed detector ("ITS3") will replace the three innermost layers of ITS2 and aims at reducing the material to the minimum by integrating a full detector half-barrel into a single piece of silicon, that will be curved around the beam pipe. The resulting layout is shown in Fig. 3, and Tab. 1 gives the main parameters.

It is worth noting, that the full detector is only connected on one side (the "A side") and that its two halves contain a single chip per layer. 


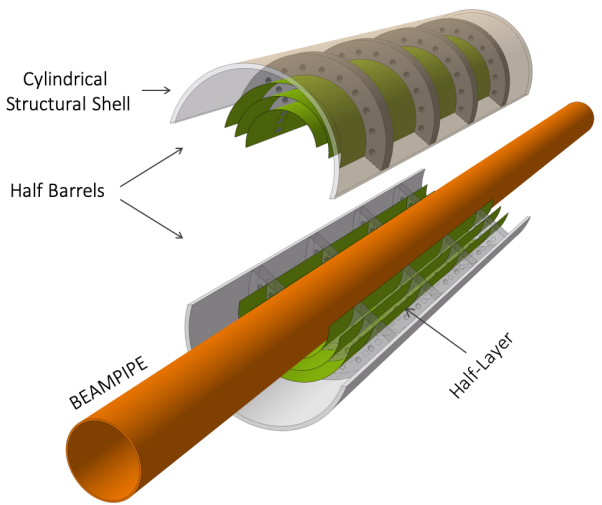

(a) separation into two half-cylinders

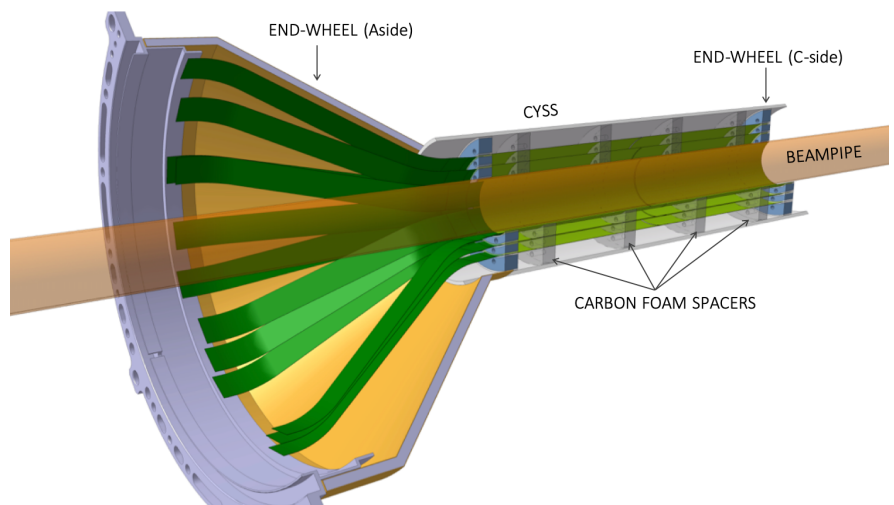

(b) detail of one half, showing the connection and routing of the services to the A-side, as well as the mechanical support of the silicon by carbon foam spacers

Figure 3: Layout of the ITS3. Figures from [3].

\subsection{Sensor chip}

Clearly, a new sensor chip will need to be developed. With the recent development of ALPIDE, the sensor chip for the ITS2, the collaboration has gained a lot of experience and insight in the technology (TowerJazz $180 \mathrm{~nm}$ ). However, due to time constraints, not all options offered by the technology, in particular stitching, could be exploited at the time of the ITS2 and will be addressed now.

- Technology As baseline, a chip in the TowerJazz $65 \mathrm{~nm}$ process will be developed. The main

Table 1: Parameters of the ITS3 layout.

\begin{tabular}{|c|c|c|c|}
\hline \multirow[b]{2}{*}{ Parameter } & \multicolumn{3}{|c|}{ Layer } \\
\hline & Layer 0 & Layer 1 & Layer 2 \\
\hline Radial position (mm) & 18.0 & 24.0 & 30.0 \\
\hline Sensitive length $(\mathrm{mm})$ & & 300 & \\
\hline Pseudo-rapidity $(\eta)$ coverage & \pm 2.5 & \pm 2.3 & \pm 2.0 \\
\hline Active area $\left(\mathrm{cm}^{2}\right)$ & 610 & 816 & 1016 \\
\hline Pixel sensor dimensions $\left(\mathrm{mm}^{2}\right)$ & $280 \times 57$ & $280 \times 75$ & $280 \times 94$ \\
\hline Number of sensors per layer & & 2 & \\
\hline Pixel size $\mu \mathrm{m}^{2}$ & & $O(10 \times 10)$ & \\
\hline Material budget $\left(\% X_{0}\right)$ & & $<0.05$ & \\
\hline Parameter & & Beampipe & \\
\hline Inner diameter (mm) & & 16 & \\
\hline Thickness $(\mu \mathrm{m})$ & & 500 & \\
\hline Material budget $\left(\% X_{0}\right)$ & & 0.14 & \\
\hline
\end{tabular}


advantages of this choice are the larger wafers ( $300 \mathrm{~mm}$ with respect to $200 \mathrm{~mm}$ at $180 \mathrm{~nm}$ ), which will allow to use a single chip to equip a full layer length, as well as a beneficial reduction of power consumption.

- Stitching Chip manufacturing is classically based on lithography that uses masks of a few square centimetres, defining the maximum size of a chip on a wafer. Several chips are placed on a wafer by stepping and exposing the same mask at different locations. "Stiching" refers to the aligned exposure of these masks down to the level of the circuit structure sizes, allowing to produce chips that are larger than the single mask, eventually reaching the size of the full CMOS wafer.

For all this to work, the chip design needs to be partitioned into a repetitive element (the pixel matrix) and a periphery to provide bias and connection to the outside. Moreover, a design for a wafer-scale chip will need to address issues of yield and power distribution, which in turn impose design rules to be met.

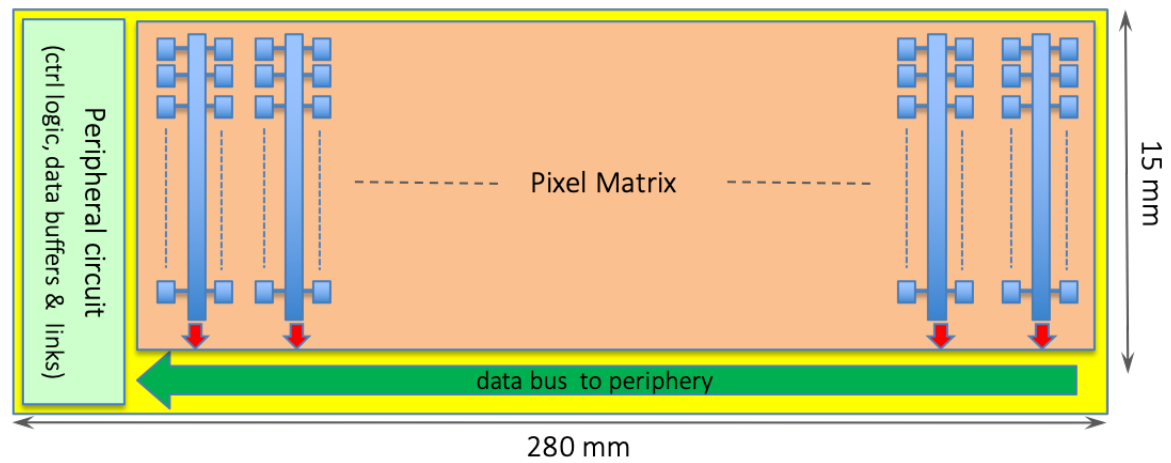

Figure 4: Possible readout architecture. Figure from [3].

As baseline, an architecture similar to ALPIDE is proposed, see Fig. 4. Depending on the detector layer, a number of these blocks will be repeated along the long edge.

\subsection{Thinning and bending}

Already when working with $50 \mu$ m-thick sensors, bending or warping is omnipresent-mostly with a negative connotation, though. Figure 5 illustrates the flexibility of the material, with Fig. 5a showing a $50 \mu \mathrm{m}$-thin flexible ALPIDE chip, and Fig. 5b showing one of the ALPIDE wafers that detached from the support during the thinning process.

Studies on several CMOS chips show that bending of thin wafers is not only mechanically, but also electrically feasible, down to bending radii much smaller than what is needed for this detector. In particular $20 \mu \mathrm{m}$-thin chips were bent to radii of far below $10 \mathrm{~mm}$ [4].

The assessment of a bent MAPS detector, especially on wafer-scale, is subject to detailed R\&D. Plans are to start from ALPIDE wafers.

\subsection{Mechanics and cooling}

The mechanical concept of the new detector is mainly based on the intrinsic stiffness of a cylindrical roll of silicon wafer. Still, to keep radii and positions constraint, the bent chips will be 


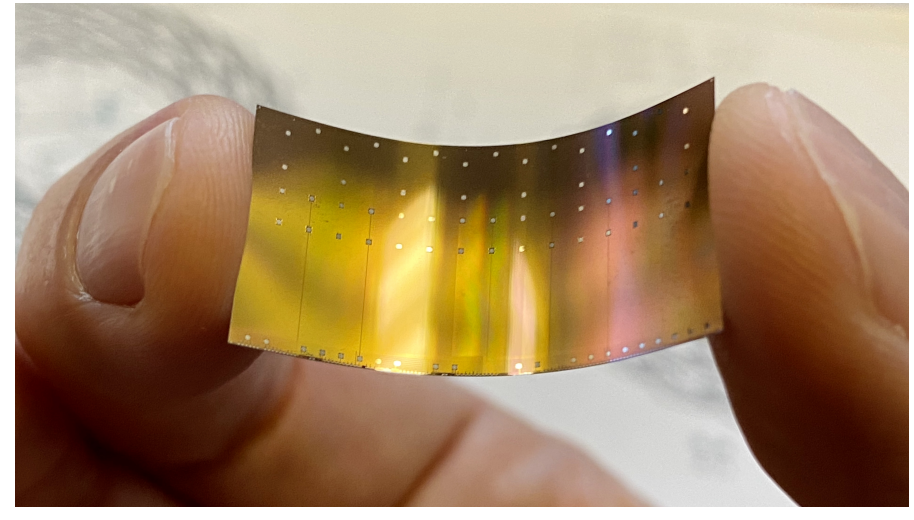

(a) A $50 \mu$ m-thick ALPIDE chip

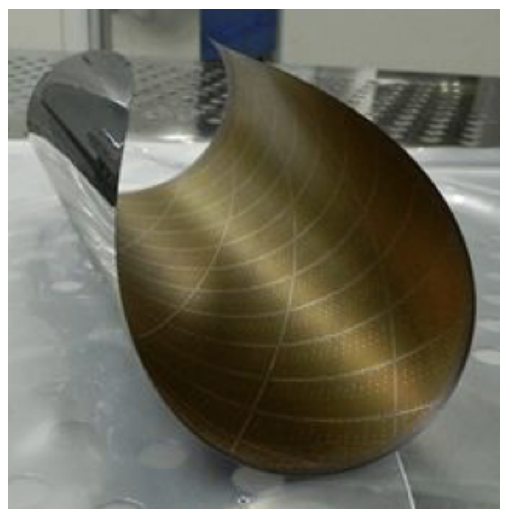

(b) An ALPIDE wafer that detached during the thinning process.

Figure 5: Bent silicon from the ALPIDE production of ITS2.

supported by open-cell carbon foam in the active area of the detector as well as with stiff support structures outside.

Cooling will be based on laminar air flow between layers in axial direction. A set of windtunnel tests is foreseen to characterise these objects mechanically and thermally and to develop a detailed understanding in terms of Finite Element Models (FEM) and Computational Fluid Dynamics (CFD).

Eventually, the integration into ALICE will amount to replacing the Inner Barrel of ITS2. This is indeed an operation whose feasibility was central to the design of ITS2, which foresees the possibility to replace (faulty) detector parts during an LHC winter shutdown.

\subsection{Beam pipe}

Finally, a new beam pipe with a wall thickness of $500 \mu \mathrm{m}$ at a radial distance covering $16 \mathrm{~mm}$ to $16.5 \mathrm{~mm}$ is foreseen. This will not only allow to place the innermost layer as close as $18 \mathrm{~mm}$ away from the nominal beam position, but will also reduce the material budget associated with the beam pipe itself (which will dominate the total, Tab. 1). This is certainly very challenging in terms of precision mechanics, and its integration (welding) will require dedicated R\&D. Moreover, since a substantial investment goes with this item, its construction will only start once the remaining ITS3 R\&D is positive.

\section{Predicted performance}

To quantify the performance boost associated with the new detector, Monte-Carlo studies were carried out to determine first basic improvements on the track reconstruction accuracy and second the associated impact on a set of key physics observables.

\subsection{Pointing resolution}

Figure 6 shows the improvement associated with ITS3 over ITS2. An improvement if a factor of 2 in the pointing resolution is present in both longitudinal and transverse directions and over a 


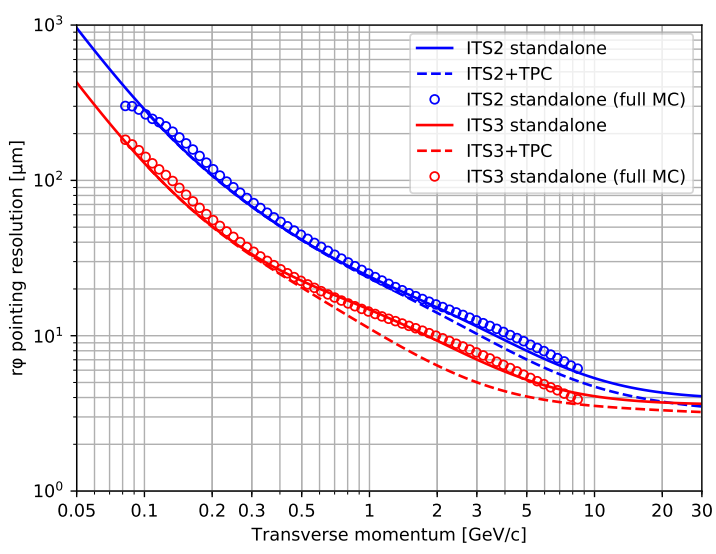

(a) in $r \varphi$ direction

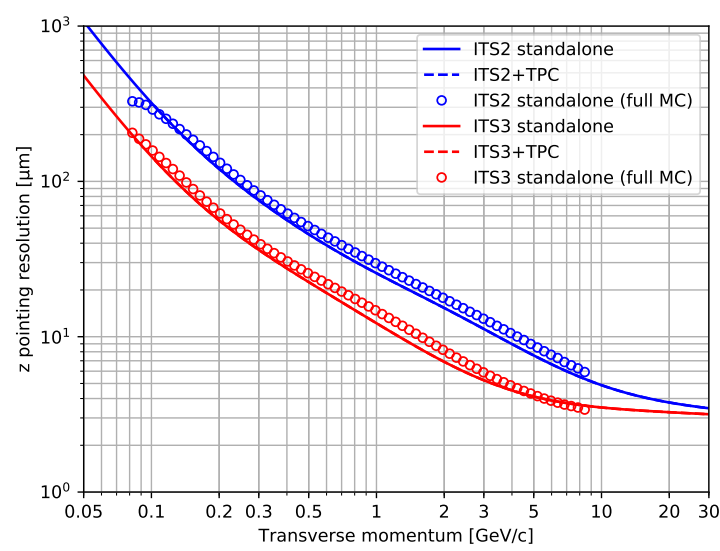

(b) in $z$-direction

Figure 6: Projected pointing resolutions of ITS2 and ITS3, Figure from [3].

large momentum range of below $10 \mathrm{GeVc}$, where closeness to interaction point and low material budget are essential. In other words, the ITS 3 will produce pictures with 4 times the resolution that ITS2 has and will hence be able to resolve structures that were smeared out before.

\subsection{Physics benchmark}

One of the strengths of ALICE with respect to the other LHC experiments is its low-momentum reach. In this regime, it is key to reduce material budget as much as possible to make a precise measurement of the particle trajectories. It is hence assumed that any such improvement will directly impact several analyses that are being carried out and, further more, will open up new channels. To quantify the effect, the reconstruction of the $\Lambda_{c}^{+} \rightarrow p K^{-} \pi^{+}$was studied in detail. The short lifetime of the $\Lambda_{c}^{+}$baryon $(\approx 60 \mu \mathrm{m})$, clearly sets a challenge to being able to separate its decay from the multitude of order of 10000 particles that are produced in a central $\mathrm{Pb}-\mathrm{Pb}$ collision at LHC energies.
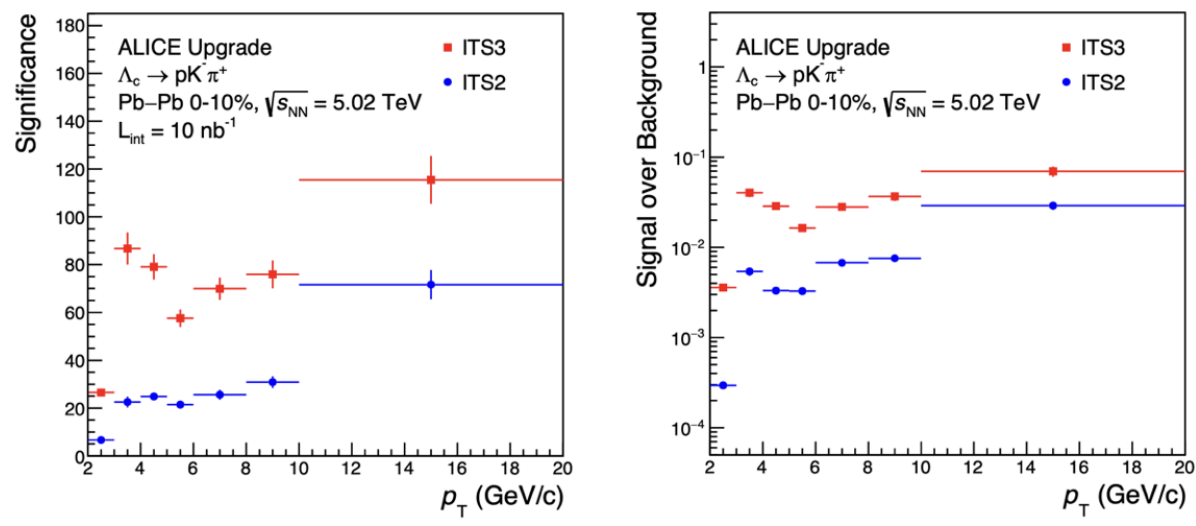

Figure 7: Performance of the $\Lambda_{c}^{+}$reconstruction, simulated with the ITS2 and the ITS3. Figure from [3].

Figure 7 shows the quantitative improvement that is expected from this new detector. One 
clearly sees that the reconstruction of a three-prongs decay benefits at higher order from the single track improvement.

\section{Summary}

The proposed detector will have unprecedented performance and will approach the limit of the mass-less detector. A big part of ALICE physics reach will significantly benefit from this improvement, and new analysis channels are expected to open up.

In its 139th meeting (September 2019) the LHCC, endorsed the start of the R\&D of this project with the goal of preparation of a Technical Design Report in time for the LHC Long Shutdown 3 [5], which the collaboration is keen to start today.

\section{References}

[1] ALICE Collaboration, Technical Design Report for the Upgrade of the ALICE Inner Tracking System, J. Phys. G41, doi:10.1088/0954-3899/41/8/087002, 2014

[2] G. Contin on behalf of the ALICE Collaboration, The MAPS-based ITS Upgrade for ALICE, this issue, 2019

[3] ALICE Collaboration, Letter of Intent for an ALICE ITS Upgrade in LS3, 2019, CERN-LHCC-2019-018, LHCC-I-034

[4] D.A. van den Ende et al., Mechanical and electrical properties of ultra-thin chips and flexible electronics assemblies during bending, Microelectron. Reliab. 54, 2014, doi:10.1016/j.microrel.2014.07.125

[5] Large Hadron Collider Committee, Minutes of the one-hundredth-and-thirty-ninth meeting held on Wednesday and Thursday, 11-12 September 2019, 2019, CERN/LHCC-2019-010, LHCC-139 